\title{
Conservation and divergence of known apicomplexan transcriptional regulons
}

Kobby Essien ${ }^{1,2}$, Christian J Stoeckert $\mathrm{Jr}^{2,3^{*}}$

\begin{abstract}
Background: The apicomplexans are a diverse phylum of parasites causing an assortment of diseases including malaria in a wide variety of animals and lymphoproliferation in cattle. Little is known about how these varied parasites regulate their transcriptional regulons. Even less is known about how regulon systems, consisting of transcription factors and target genes together with their associated biological process, evolve in these diverse parasites.

Results: In order to obtain insights into the differences in transcriptional regulation between these parasites we compared the orthology profiles of putative malaria transcription factors across species and examined the enrichment patterns of four binding sites across eleven apicomplexans.

About three-fifths of the factors are broadly conserved in several phylogenetic orders of sequenced apicomplexans. This observation suggests the existence of regulons whose regulation is conserved across this ancient phylum. Transcription factors not broadly conserved across the phylum are possibly involved in regulon systems that have diverged between species. Examining binding site enrichment patterns in light of transcription factor conservation patterns suggests a second mode via which regulon systems may diverge - rewiring of existing transcription factors and their associated binding sites in specific ways. Integrating binding sites with transcription factor conservation patterns also facilitated prediction of putative regulators for one of the binding sites.
\end{abstract}

Conclusions: Even though transcription factors are underrepresented in apicomplexans, the distribution of these factors and their associated regulons reflect common and family-specific transcriptional regulatory processes.

\section{Background}

The apicomplexans are a phylum of about 5000 protozoan organisms that parasitize almost all animals [1]. Despite sharing three invasion-related organelles, collectively called the apical complex, these organisms are diverse and pursue a wide variety of lifestyles that result in varied diseases of medical and veterinary importance. Plasmodium species are malaria-causing mosquitoborne parasites. Theileria species are tick-transmitted parasites that cause lymphoproliferative diseases in cattle. Cryptosporidium species do not require a vector and are spread from host-to-host causing cryptosporidiosis, an acute diarrheal disease in mammals.

Sequencing and subsequent analysis of the human malaria parasite Plasmodium falciparum revealed that the parasite has few known or putative transcription

\footnotetext{
* Correspondence: stoeckrt@pcbi.upenn.edu

${ }^{2}$ Center for Bioinformatics, University of Pennsylvania, 1420 Blockley Hall, 423 Guardian Drive, Philadelphia, Pennsylvania 19104, USA
}

factors [2,3]. A similar dearth of transcription factors has been observed in other apicomplexans [4,5]. Even with the discovery of the existence of a family of about 26 AP2 transcription factors in these parasites [6], compared to other single-celled eukaryotes such as yeast, apicomplexans still have a paucity of DNA-binding transcription factors.

In their analysis of Plasmodium noncoding regions, Imamura and colleagues identified three putative binding sites in rodent parasites [7]. We recently, described seven putative transcription factor binding sites that were conserved in at least two Plasmodium species [5]. Three of these binding sites were broadly conserved across human, primate and rodent malaria parasites. Other work has described the conservation of binding sites across Theileria [8] and Cryptosporidium species [9]. These results have raised the question of how broadly conserved the control of transcription is across apicomplexans. Specifically, is transcriptional control 
largely conserved across apicomplexans or are transcription factors and their binding sites in promoters of target genes only conserved between closely related subsets of apicomplexans?

Differences in transcriptional control are thought to contribute to species diversity [10]. High throughput genomics approaches have highlighted divergence of known binding sites of conserved transcription factors in related yeast species [11] and between human and mouse [12]. It has been noted that the P. falciparum AP2 transcription factor PF14_0633 and its Cryptosporidium parvum ortholog, cgd2_3490, likely regulate different sets of genes [13]. The nucleotide word TGCATGCA, identical to the PF14_0633 binding site, is overrepresented in all sequenced apicomplexan genomes except Theileria $[14,15]$. This suggests that the diversification principles at work in apicomplexa may be similar to those at work in other eukaryotes.

Despite belonging to the same phylum, apicomplexans are characterized by a striking amount of diversity. Members of the blood-invading haemosporidian order of parasites include the mosquito-transmitted Plasmodium species that cause malaria. Even though Theileria species also invade red blood cells, they belong to the piroplasmid order of apicomplexans and are transmitted by ticks and mostly cause lymphoproliferative diseases in cattle. Then there are Toxoplasma and Neospora both of which fall in the eucoccidioridian order and do not require a host and exhibit very little cell tropism. The issue of whether these parasites modulate their transcriptional control to pursue such extremely diverse lifestyles and inhabit distinct niches remains largely unexplored.

In this work, we examined the conservation of putative Plasmodium falciparum transcription factors across apicomplexans. We discover that disparate subsets of apicomplexans likely have different sets of transcription factors suggesting divergence in the complements of factors present in these species. Additionally, our analysis reveals that about three-fifths of putative Plasmodium falciparum transcription factors have an ortholog in at least one non-Plasmodium apicomplexan. Enrichment analysis of previously identified promoter binding sites across species also suggests large-scale differences in transcriptional control in disparate apicomplexans. None of four transcriptional regulons considered in detail were conserved across all apicomplexa. However, the conservation of two regulons spanned blood-invading phylogenetic orders within the phylum. By matching the conservation profiles of putative transcription factors with the enrichment profiles of binding sites we were able to predict candidate transcription factors for a previously partially characterized ribosomal regulon. Finally, by integrating the conservation analysis of transcription factors with the enrichment analysis of binding sites we detected two possible mechanisms via which regulons may be modified in these parasites.

\section{Results}

In its simplest form, a transcriptional system for a regulon can be reduced to three components: a transcription factor, the set of target genes or regulon identifiable by an upstream binding site that is bound by the transcription factor, and the biological process connected to the regulon. We pursue complementary approaches to assess whether regulons differ between apicomplexans. The first approach entails determining whether transcription factors from the most widely studied apicomplexan, $P$. falciparum, are conserved in other apicomplexans. In the second approach, we seek to determine whether four previously experimentally characterized transcription factor binding sites from $P$. falciparum and $P$. berghei are conserved (enriched) upstream of similar genes in different parasites.

\section{Conservation patterns of putative $P$. falciparum transcription factors}

The well-known diversity of lifestyles of disparate apicomplexans as well as the age of the phylum (700-900 million years [16]) led us to hypothesize that significant differences exist in the sets of transcription factors present in each species. To test this hypothesis we generated a list of putative $P$. falciparum transcription factors by identifying all $P$. falciparum proteins containing known DNA-binding domains and examined the conservation patterns of these proteins across three disparate sets of apicomplexans (Figure 1). We limit ourselves to domains possibly involved in sequence-specific DNA-binding, consequently our list of predicted $P$. falciparum transcription factors (Additional File 1) is smaller than the list of transcription associated proteins assembled by Coulson and colleagues in their comparison of transcriptional control between $P$. falciparum and other eukaryotes [3].

To establish true orthology between two genes, one would have to perform a phylogenetic study and demonstrate that a pair of genes descended from a common ancestor. This would be followed up with biological validation that the two genes do in fact have the same function in each of the two species. However detailed studies of that nature are not available for most apicomplexan genes. Consequently, we considered putative orthologs established by two commonly utilized methods i) orthology prediction via reciprocal best BLAST hits (RBH) between species and ii) orthology prediction obtained from OrthoMCL, which couples reciprocal best BLAST hits with protein clustering and offers additional refinements to orthology prediction 


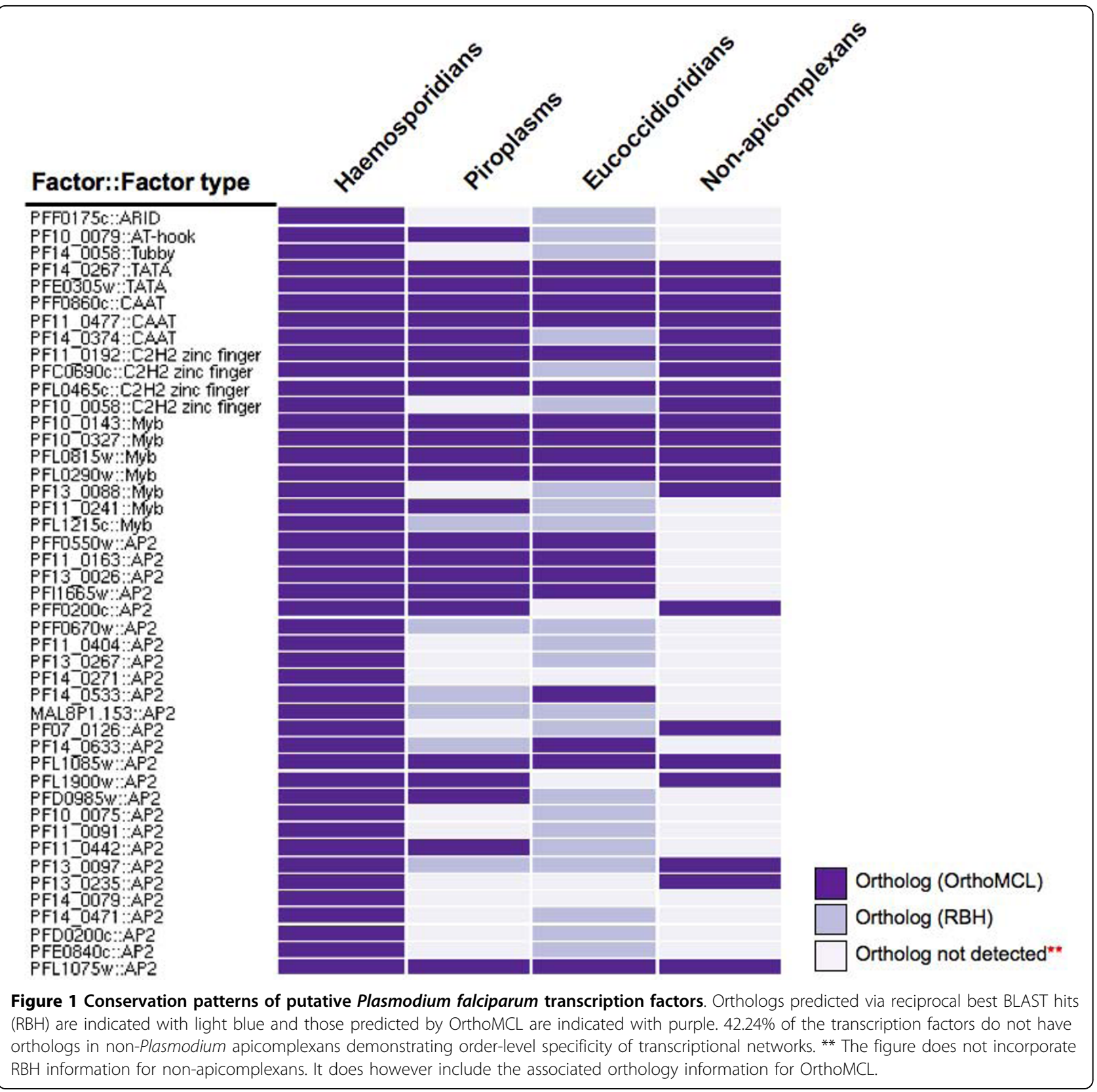

including detecting co-orthologs and accounting for genome-wide base composition biases [17].

Initial analyses using the reciprocal best BLAST hits $(\mathrm{RBH})$ method for ortholog identification revealed that not all transcription factors are conserved across the apicomplexan phylum (Figure 1). In order to make stronger claims about the conservation (or lack thereof) of transcription factors, we reran the analyses using data from OrthoMCL. In all subsequent analyses, we rely on OrthoMCL for ortholog predictions because of the aforementioned advantages that OrthoMCL has over the reciprocal best BLAST hits approach.
Analysis of putative transcription factors by OrthoMCL revealed that essentially all $P$. falciparum predicted transcription factors have orthologs in at least one other Plasmodium species (haemosporidians) (Figure 1). However, only $57.76 \%$ of $P$. falciparum predicted factors have an ortholog in at least one non-Plasmodium apicomplexan (piroplasms and eucoccidioridians). To determine whether transcription factors from other apicomplexans also have similar numbers of orthologs in P. falciparum, we predicted transcription factors in other apicomplexans using the methodology used for predicting $P$. falciparum transcription factors. We then 
checked for the presence of orthologs of these factors in $P$. falciparum. On average, $94.13 \%$ of primate-infecting $P$. vivax and $P$. knowlesi factors are conserved in $P$. falciparum, as are $78.91 \%$ of the factors from the rodent malaria parasites $P$. berghei and $P$. yoelii. The piroplasms T. annulata and T. parva have on average $33.44 \%$ of their predicted factors present in P. falciparum while the eucoccidioridians $T$. gondii and $N$. caninum have only $15.42 \%$. Finally, C. parvum has $28.57 \%$ of its predicted factors present in P. falciparum. Lists of the predicted transcription factors in the other species are presented in Additional File 1.

The level of conservation of transcription factors between $P$. falciparum and the non-Plasmodium species was surprising given the age of the phylum. In an examination of fungus-specific predicted transcriptional regulators it was noted that the budding yeast Saccharomyces cerevisiae and the fission yeast Schizosaccharomyces pombe, which are separated by 400-600 million years of evolution [18], share only $15 \%$ of fungal-specific regulators [19]. While we cannot directly compare data from the aforementioned yeast study to ours as different criteria for factor prediction were utilized, we did not expect to see extremely high levels of conservation among the parasite species under consideration as they belong to a phylum estimated to be 700-900 million years old [16].

\section{Assessing conservation of $P$. falciparum merozoite invasion, ookinete stage, ribosomal gene, and sporozoite stage regulons across apicomplexan species}

We further tested the hypothesis that transcriptional regulons differ significantly between apicomplexans by examining whether transcription factor binding sites were conserved (enriched) upstream of the same sets of genes in eleven apicomplexan species. Hits or targets of a transcription factor were identified as those genes with a high confidence instance of the associated binding site in their 2000 bp upstream region (for details see Figure 2 and Methods).

We examined the conservation of regulons associated with four binding sites in particular (Figure 3). We focus on these sites as they are well described in the literature. De Silva and colleagues used protein binding microarrays (PBM) to show that the first and third sites in Figure 3 are bound by the AP2 transcription factor PF14_0633 [13,20] and PFF0200c [13] respectively (the functionality of the third site had been previously established by Voss et al. [21]). The transcription factor binding to the second site is unknown but the functionality of the site has been determined via deletion assays by Militello et al. [22]. Using chromatin immunoprecipitation (ChIP) and electromobility shift assays (EMSA), Yuda et al. [23] recently confirmed PF-O
(PB000572.01.0) as the transcription factor for the fourth site.

For each of the four binding sites, a regulon was identified as conserved in a species if its known experimentally-associated transcription factor (See Figure 3) was present (as an ortholog) in the species and hits for the site have statistically significant overlap (enrichment) with the set of genes expected to be regulated by the site (expected regulon).

\section{Cell invasion regulon}

Conservation of enrichment of the merozoite cell invasion binding site in Plasmodium species and T. annulata only suggests a divergence in this system among members of the phylum (Figure 4). Curiously, the cell invasion binding site was not conserved in T. parva and $B$. bovis. Generally, the trend of $p$-values suggests a greater extent of enrichment in T. parva and B. bovis than in $T$. gondii, N. caninum and C. parvum.

We examined the conservation pattern of the cell invasion site's known regulator across sequenced apicomplexans. The AP2 transcription factor PFF0200c had orthologs in Plasmodium species, the two Theilerias and Babesia suggesting that the factor is conserved across the aforementioned species (Figures 1 and 4). Conservation of the factor in all the piroplasms with enrichment for the site in only one of the three piroplasms suggests partial conservation of the regulon in piroplasms. The lack of significant enrichment $p$-values and absence of PFF0200c orthologs in T. gondii, N. caninum and $C$. parvum suggests that this regulon is not conserved in eucoccidioridians.

\section{Ookinete regulon}

The ookinete is an invasive stage of Plasmodium parasites within the mosquito. Enrichment for the ookinete binding site is seen only in Plasmodium species. The orthology analysis suggested that the factor is conserved in all Plasmodium species (Figure 4).

\section{Ribosomal regulon}

The G-box binding site is not enriched upstream of ribosomal genes across all sequenced apicomplexans. This binding site is generally conserved upstream of ribosomal genes in Plasmodium species, the two Theilerias and B. bovis (Figure 4). As in the case of the cell invasion and ookinete sites, this ribosomal binding site is not conserved in $T$. gondii, N. caninum and $C$. parvum.

The transcription factor associated with the G-box has not been identified so its orthology pattern could not be assessed. As seen in Figure 1, eight putative transcription factors have similar orthology patterns as the G-box - absent in all the three eucoccidioridians and 


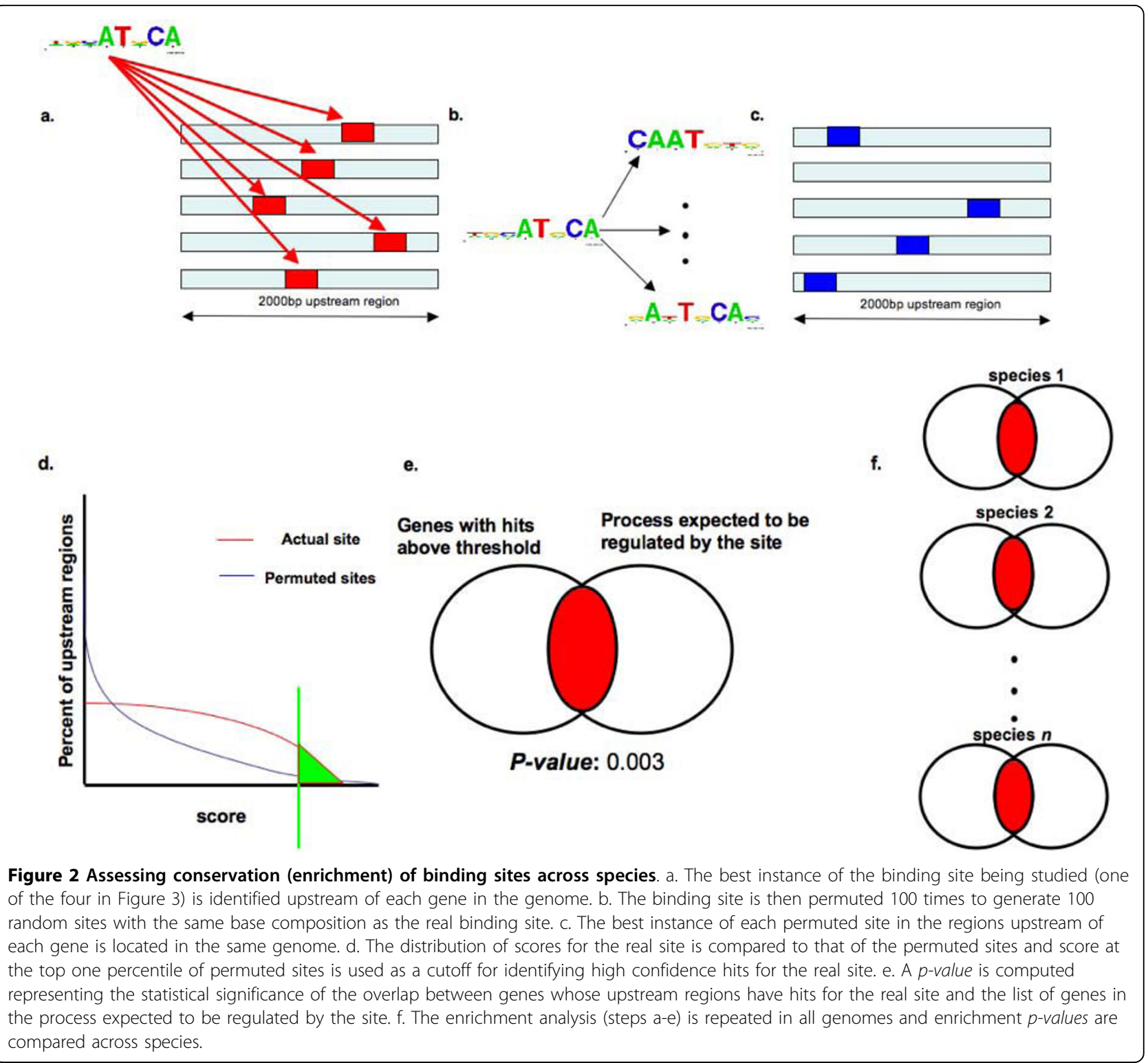

present in some haemosporidians and some piroplasms. Any of these eight proteins (Additional File 2) may be negative or positive regulators of G-box associated ribosomal genes. One of the eight the proteins, AP2 protein PFF0200c, was ruled out as a regulator for these genes as it binds to the cell invasion site identified by De Silva et al. [13] and is also not enriched upstream of ribosomal genes in any of the species considered (Table S6 in Additional File 3). Additionally, another of the eight proteins is predicted to be a CAAT-binding factor (Additional File 2) consequently, it too is unlikely to bind to the G-box.

To further explore the possibility that the remaining six proteins regulate the ribosomal genes in question, we compared the expression patterns of these candidate regulators to those of ribosomal genes with G-box hits (these high confidence putative G-box hits are presented in Additional File 4). Of these six, two are positively correlated with the average profile of G-box associated ribosomal genes and the other four are negatively correlated (Additional File 2). The protein with the highest positive correlation $(r=0.651)$ and hence the highest-ranking candidate positive regulator is the $\mathrm{C} 2 \mathrm{H} 2$ zinc finger protein PFC0690c. The protein with the highest negative correlation $(r=-0.838)$ and hence the highest-ranking candidate negative regulator is the AP2 protein PF11_0442. Figure 5 shows that the average profile of the G-box associated ribosomal proteins lags the profiles of both these high-ranking candidate regulators. 


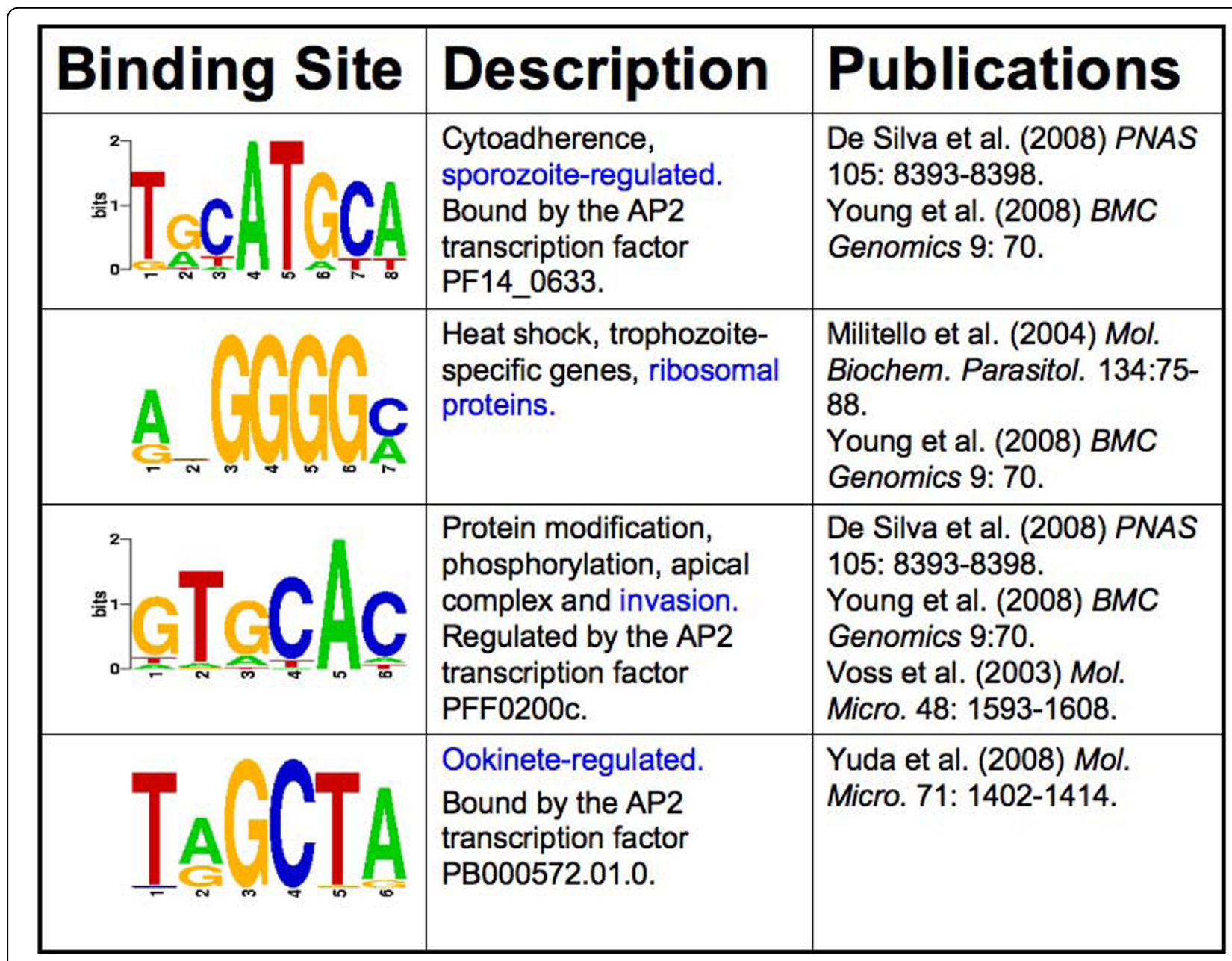

Figure 3 Transcription factor binding sites considered in this study. The functional contexts considered in the study are highlighted in blue. The transcription factor for the site, if known, and key publications are also presented.

\section{Sporozoite regulon}

This regulon is conserved only in Plasmodium species suggesting that its role in these species is quite specific (Figure 4). Its associated regulator PF14_0633 has orthologs in all other Plasmodium species and C. Parvum.

A putative model of ribosomal gene regulation in apicomplexans

In our earlier analysis of ribosomal genes, we observed that the G-box was not enriched upstream of T. gondii, $N$. caninum and C. parvum ribosomal genes. The binding site associated with $P$. falciparum sporozoite genes has been independently implicated in the regulation of T. gondii ribosomal genes [24]. We tested the hypothesis that this same binding site is enriched upstream of ribosomal genes in $N$. caninum, which is closely related to T. gondii, and to C. parvum which though separate from all the other apicomplexans considered is classed together with $T$. gondii and $N$. caninum as an eucoccidioridian. This hypothesis was found to be true in N. caninum but not in C. parvum (p-values of 0.010 and 0.960 respectively). The TRP-1 putative binding site identified by Van Poppel and colleagues upstream of $T$. gondii ribosomal genes [24] is also associated with $N$. caninum ribosomal genesbut not C. parvum ones ( $p$ values of $1.7^{*} 10^{-19}$ and 0.202 ). The sequence logo of the TRP-1 site is 'starred' with yellow in Figure 6.

These results enable us to begin assembling a model describing the regulation of ribosomal genes in apicomplexans (Figure 6). In most Plasmodium species, Theileria and Babesia these genes are possibly regulated by one of six candidate regulators mentioned earlier and presented in Additional File 2 (or their respective orthologs in the appropriate species) and the G-box binding site originally identified in P. falciparum. In T. gondii and the related parasite $N$. caninum these same genes are regulated by a factor similar to the $P$. falciparum AP2 transcription factor PF14_0633 and the site 


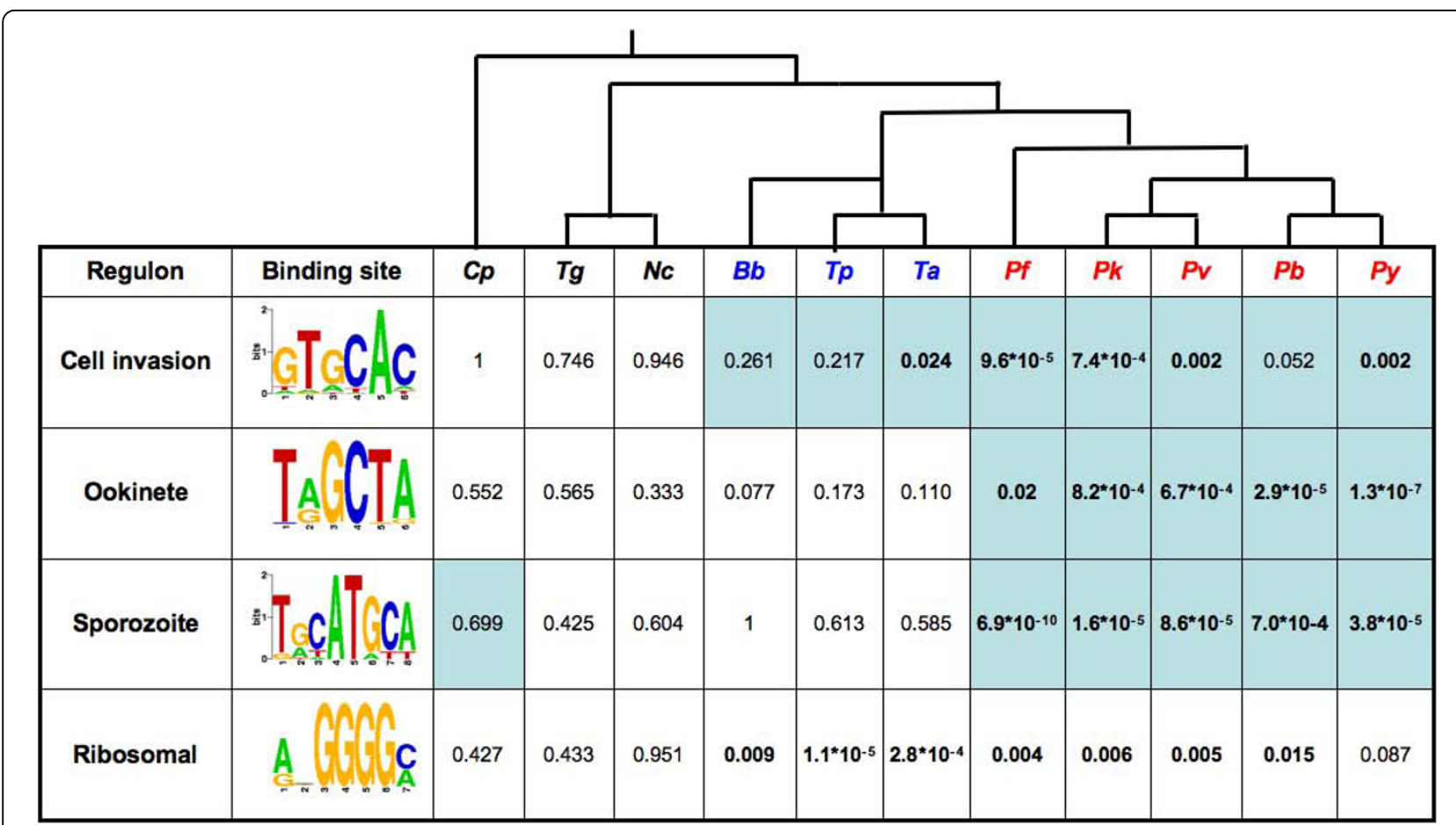

Figure 4 Conservation of the four regulons. Regulons have generally diverged across the apicomplexan phylum but genera-related conservation patterns are evident. This table presents enrichment $p$-values for each of the four regulons (rows) in each of the eleven species studied (columns). Species names (column headings) are abbreviated as presented in Table 1. Significant enrichment $p$-values are given in boldface. If the known factor for a particular regulon is conserved in a species (as determined in earlier analysis of conservation of patterns of putative transcription factors) we highlight the cell for that entry in light blue.

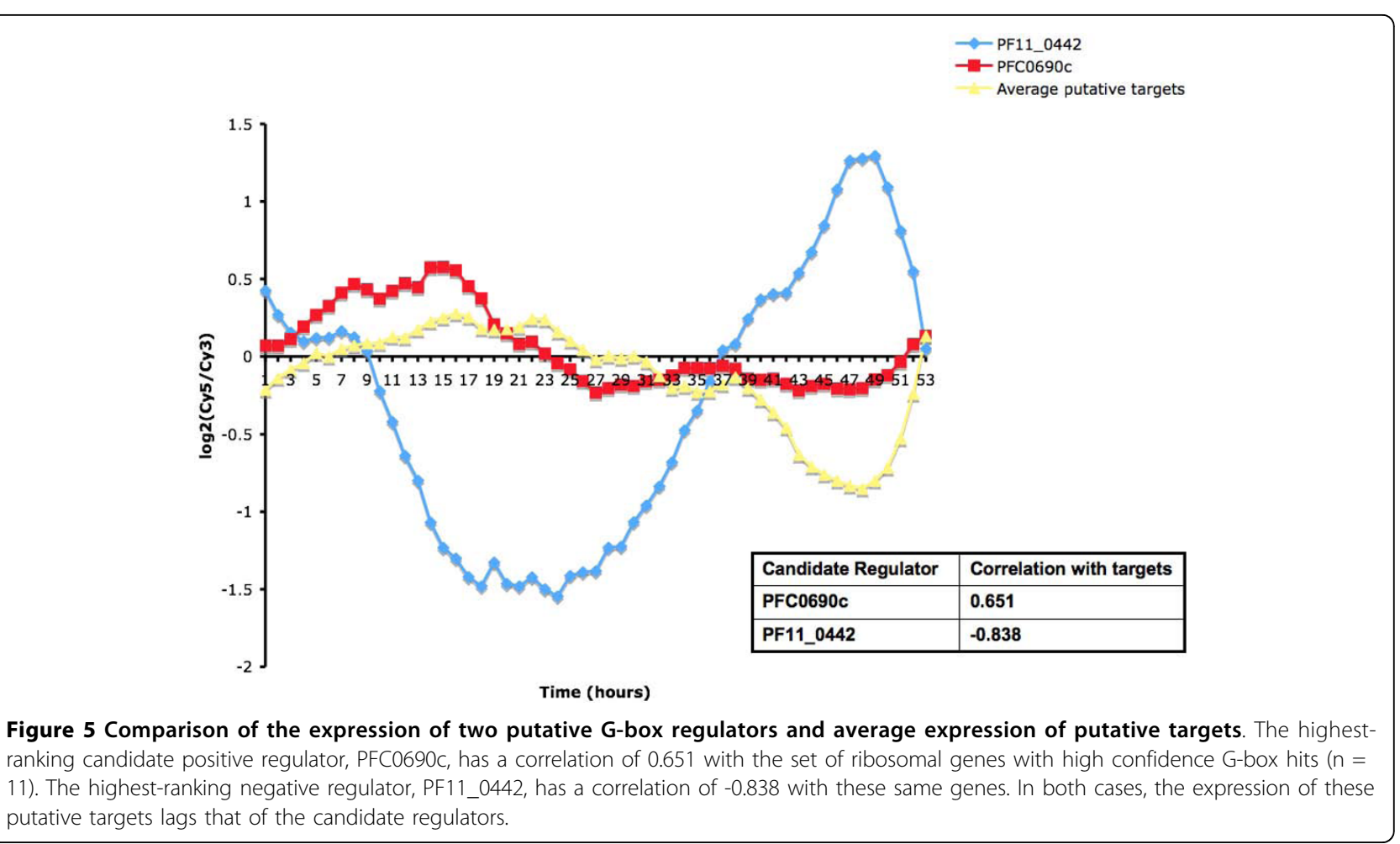




\begin{tabular}{|c|c|c|c|c|c|c|c|c|c|c|}
\hline$C p$ & $T g$ & Nc & $B b$ & $T p$ & $T a$ & $P f$ & $P k$ & $P v$ & $P b$ & $P y$ \\
\hline 0.427 & 0.433 & 0.951 & 0.009 & $1.1 * 10^{-5}$ & 0.0003 & 0.004 & 0.006 & 0.005 & 0.015 & 0.087 \\
\hline 0.960 & 0.036 & 0.010 & 1 & 0.559 & 1 & 0.995 & 0.994 & 0.933 & 0.025 & 0.352 \\
\hline 0.202 & $1.6^{\star} 10^{-15}$ & $1.7^{\star} 10^{-19}$ & 1 & 0.365 & 1 & 0.824 & 1.000 & 0.455 & 0.574 & 0.398 \\
\hline
\end{tabular}

b.

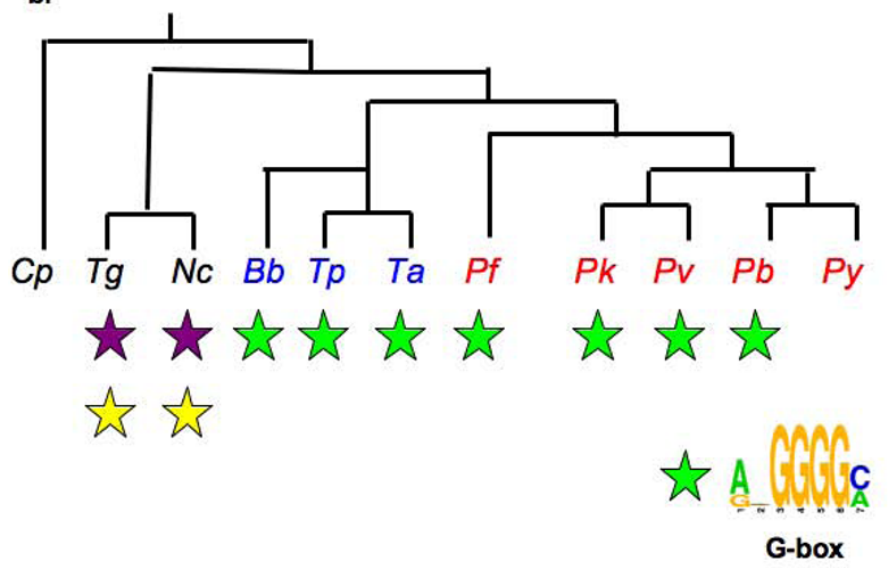

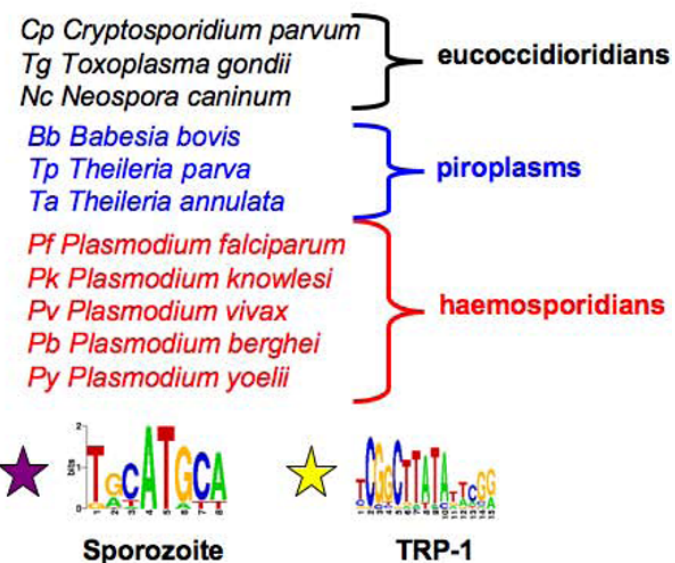

Figure 6 The ribosomal regulon system in apicomplexans. a) Enrichment $p$-values of three binding sites upstream of ribosomal genes. Stars are color-coded to represent the three transcription factor binding sites at the bottom of the image. The G-box, sporozoite and TRP-1 sites are 'starred' with green, purple and yellow respectively. b) Schematic phylogeny of apicomplexan species considered. Stars below a particular species indicate that the corresponding binding site likely regulates ribosomal genes in that particular species.

originally associated with $P$. falciparum sporozoite genes together with the TRP-1 site identified by Van Poppel and colleagues [24].

\section{Discussion}

In this work we take advantage of the availability of eleven apicomplexan genomes to examine the conservation of transcriptional regulons in parasites. We compared the conservation profiles of predicted Plasmodium transcription factors in disparate apicomplexans to obtain a regulator-centric view of differences in regulons. We also focus on regulon systems associated with four Plasmodium binding sites and compare and contrast the conservation of these regulons within the Plasmodium genus and in other apicomplexans. Overall, we note large-scale differences in the regulation of regulons as determined by different sets of putative transcription factors in these species. Additionally, in our analysis of binding sites, we note that even when a site is conserved in a particular species, it may be rewired to control a new regulon.

\section{Conservation and divergence of transcription factors}

About three-fifths of Plasmodium falciparum predicted transcription factors are conserved in at least one of the other non-Plasmodium apicomplexans considered.
Additionally, when orthologs of predicted transcription factors from other apicomplexans were sought in P. falciparum we noted that generally the further away evolutionarily a species was from P. falciparum the smaller the number of detected orthologs. This suggests that over large evolutionary distances the regulators that direct regulons are generally not conserved.

P. falciparum transcription factors that have orthologs in non-Plasmodium apicomplexans raise the interesting possibility that some transcription factors may be broadly conserved across apicomplexans. These factors may be involved in ancient apicomplexan transcriptional networks. Such factors include TATA-binding proteins and CAAT-box binding transcription factors which are well known general transcription factors, some of which have orthologs in species outside the apicomplexan phylum. These broadly conserved transcription factors also contain Myb, C2H2 zinc finger and AP2 transcription factors which may be involved in core processes a little more specialized than those regulated by TATA-binding and CAAT-binding transcription factors.

\section{Conservation of the cell invasion and ookinete regulons} Analysis of the Plasmodium falciparum cell invasion and Plasmodium berghei ookinete transcription factor 
binding sites suggests that their associated regulons are conserved across Plasmodium species. Interestingly, the cell invasion site was observed to be enriched upstream of Theileria annulata cell invasion genes as well but not those of the related parasites Theileria parva and Babesia bovis.

The transcription factor associated with the cell invasion site is conserved in all the Plasmodium species and all the piroplasms considered. It is possible that the cell invasion regulon has diverged in T. parva and $B$. babesia and the conserved transcription factor has been rewired to serve other regulatory purposes. Alternatively, the lack of enrichment of the cell invasion site in T. parva and B. bovis may reflect the low number of piroplasm genes with orthologs to known Plasmodium falciparum cell invasion genes (Table S1 in Additional File 3). Efforts to better annotate piroplasm genes will likely provide more comprehensive lists of invasion genes specific to these organisms. It will be interesting to determine whether these species-specific invasion genes are also under the control of the $P$. falciparum invasion binding site or another binding site altogether.

The cell invasion binding site, which we demonstrated was conserved in all Plasmodium species, is utilized in additional exclusive ways in $P$. falciparum. A combination of affinity purification and mass spectrometry has been used by Voss and colleagues to validate the cell invasion binding site, which they refer to as SPE2, in the upstream regions of a subset of $P$. falciparum var genes [21]. Var genes are important parts of the pathways that $P$. falciparum uses to evade the human immune system. Var genes are specific to P. falciparum so the presence of the cell invasion site in var gene upstream regions is a species-specific innovation.

In contrast to the cell invasion binding site, the ookinete site is conserved only in Plasmodium species (Figure 4). In this case, the factor is conserved across all Plasmodium species, with no conservation observed in any of the piroplasms.

\section{Transcriptional regulation of ribosomal genes in apicomplexans}

Plasmodium species and the piroplasms share a ribosomal gene regulon. In addition to controlling ribosomal genes, the binding site associated with this regulon is suspected to control other trophozoite-specific metabolic processes [25]. The observation that Theileria species possess a binding site similar to the G-box binding site identified in $P$. falciparum was first made by Guo and Silva [8]. We show that upstream of ribosomal genes, the G-box is conserved across five Plasmodium species and three piroplasms but not in the three eucoccidioridian species considered.
The regulation of ribosomal genes illustrates the complexity of transcriptional rewiring in apicomplexans (Figure 6). The G-box was conserved in Plasmodium species and piroplasms but not in eucoccidioridians. Upstream regions of ribosomal genes in the eucoccidioridians, $T$. gondii and $N$. caninum, are enriched for the binding site that was originally associated with Plasmodium sporozoite genes and a second site, TRP-1, that is not enriched upstream of the orthologous genes in the other apicomplexans. Interestingly, none of the three aforementioned ribosomal gene binding sites are enriched upstream of $C$. parvum's ribosomal genes implying the existence of disparate but yet unknown transcription factor binding sites for this parasite's ribosomal genes.

Divergence of the binding sites governing ribosomal gene regulation despite the core and conserved nature of these genes in species is not unprecedented. The upstream regions of Saccharomyces cerevisiae ribosomal genes are enriched for the RAP1 binding site but this particular site is not enriched in the upstream regions of the corresponding genes in Schizosaccharomyces pombe [26]. While both S. cerevisiae and Schizosaccharomyces pombe have copies of the RAP1 transcription factor, the S. cerevisiae copy has a transactivation domain that the Schizosaccharomyces pombe copy lacks suggesting that the factor and its corresponding site are rewired together. Additionally, the upstream regions of mitochondrial ribosomal genes in yeast species exhibiting a preference for aerobic respiration are enriched for the RGE transcription factor binding site while no enrichment is seen upstream of the same genes in yeast species with a preference for anaerobic respiration [27].

\section{Evolution of a sporozoite regulon via rewiring of binding sites}

A phylogenetic order-specific system is involved in sporozoite gene regulation in Plasmodium species. This suggests that systems governing the control of sporozoite gene expression differ between Plasmodium species on one hand and the rest of the apicomplexans on the other.

The binding site originally associated with the Plasmodium sporozoite regulon described above has different roles in other apicomplexans suggesting that it illustrates a case where a system evolves by rewiring of sites in different species. Specifically, the site is enriched upstream of ribosomal genes in the two eucoccidioridians $T$. gondii and $N$. caninum. Additionally, others have suggested that the transcription factor associated with the site is in fact conserved in all apicomplexans and also that this factor is associated with different sets of genes in Plasmodium falciparum and Cryptosporidium parvum [13]. It is worth noting that this factor is likely multifunctional even in Plasmodium species, as it 
has been demonstrated to be active in Plasmodium red blood cell and sporozoite stages $[13,28]$.

\section{A role for adaptive evolution in the conservation patterns of transcriptional regulons in apicomplexans?}

Differences in the conservation of putative transcription factors and known binding sites suggest that regulons have diverged between apicomplexan species. A natural question that arises from the results of this study is whether the observed differences are the result of neutral or adaptive evolution.

Under the neutral evolutionary scenario, over evolutionary time, orthologous transcription factors have accumulated so many mutations, albeit neutral ones, that though their functions may remain the same they are no longer identifiable as orthologs by computational means.

While experimental evidence for adaptive changes in transcription factors is not available for apicomplexans, there is computational evidence to suggest that such adaptation is highly likely. In higher eukaryotes, coding regions of transcription factors have been observed to diverge more quickly than those of genes involved in housekeeping processes like metabolism [29]. In a study of five Plasmodium species we noted that Plasmodium transcription factors and parasite lifestyle-associated genes might behave similarly as they have less constrained coding regions (higher $\mathrm{dN} / \mathrm{dS}$ or rates of nonsynonymous substitutions per nonsynonymous site to synonymous substitutions per synonymous site) than other groups of genes [30].

The cell invasion, ookinete and sporozoite binding sites all have some Plasmodium-specific conservation patterns. Though instances of these sites are present in all the apicomplexan species considered, they all have sets of genes involved in a biological process or lifecycle stage whose upstream regions are enriched for these sites in Plasmodium species only (in the case of the cell invasion site we see association in one of the three piroplasms as well). The ribosomal genes have a specific binding site for Plasmodium species and piroplasms and another set of binding sites for $T$. gondii and N. caninum. Such changes in regulons are not unprecedented. In a similar study of yeast species separated by 420-600 million years of evolution only one out of forty-two regulons was conserved across fourteen species considered. Additionally, more distantly related yeast species shared fewer binding sites [31].

The lack of conservation of binding sites in all apicomplexans could result from neutral evolution - over 700-900 millions years of apicomplexan evolution a remarkable amount of drift is expected in the upstream regions of distantly related parasites.
Alternatively, these regulon systems might be evolving adaptively in response to genus-related lifestyle and environmental differences. This is possible given that all the binding sites considered were correlated with specific genera or groups of genera within the phylum. However, further work is necessary to distinguish between these two scenarios. Probabilistic methods exist for examining the types of selection, if any, on binding sites within closely related organisms - yeast species $(20$ million years of separation) and fly species (10 million years of separation) - however these methods require high quality alignments $[32,33]$ which are not easy to generate for the distantly related apicomplexans in our study.

\section{Conclusions}

In this work, we demonstrate the power of comparative genomics in shedding light on transcriptional regulation in non-model genomes with little prior knowledge. We integrate eleven apicomplexan genomes with observations from high-throughput regulation studies and highlight different modes of transcriptional regulon modification. While transcription factors are underrepresented in these parasites it appears that these species use the few factors they have in phylogenetic order-specific ways. As more annotation, expression data and results from experimental studies of transcription factors in these parasites become available it will be possible to develop a more global perspective of transcriptional differences, and their functional consequences if any, in these important parasites.

\section{Methods \\ Orthology}

Orthologs were predicted via two methods. The first method is referred to as the reciprocal best BLAST hits (RBH) approach. Under the RBH framework, orthologs were determined by identifying reciprocal best BLAST hits between various apicomplexans. An E-value cutoff of $10^{-5}$ was utilized. OrthoMCL implements a more rigorous approach for the identification of orthologs. OrthoMCL first identifies reciprocal best BLAST hits between species and reciprocal 'better' BLAST hits within species. It utilizes an E-value cutoff of $10^{-5}$. Markov Clustering (MCL) is then used to better define the orthology and paralogy relationships between proteins from the resulting graph of BLAST E-values [17,34].

Orthologs were primarily obtained during the course of this work from two runs (versions 2.1 and 3.0) of OrthoMCL-DB [17] kindly provided by Deborah Pinney. OrthoMCL version 2.1 covering 89 genomes was used for the identification of orthologous apicomplexan upstream regions. The Babesia bovis genome is currently not included in OrthoMCL-DB so P. falciparum $B$. bovis orthologs were obtained from a separate run of the OrthoMCL algorithm kindly provided by Kuo and 
colleagues [16]. For more comprehensive analysis of orthologous transcription factors, OrthoMCL version 3.0 covering 128 genomes was used for the transcription factor orthology analysis. In one case, OrthoMCL 3.0 did not identify any haemosporidian orthologs for a $P$. falciparum factor (MAL8P1.153), but examination of syntenic regions revealed that orthologs do in fact exist in the two other primate malaria parasites. Apicomplexan orthologs for the 45 P. falciparum predicted transcription factors are presented in Additional File 5.

\section{Identification of putative transcription factors}

Known DNA-binding domains $[3,35]$ were obtained from version 23.0 of the Protein families database (Pfam) [36]. Hidden Markov Model representations of these domains were scanned against apicomplexan proteomes to identify putative transcription factors. A protein is defined as containing a domain if its E-value exceeds the domainspecific Pfam-defined gathering threshold. The gathering threshold of a specific Pfam Markov Model is the sequence-based cutoff at which the Pfam curators "gather" sequences to include in that Markov Model. 45 putative transcription factors were identified in P. falciparum. The predicted transcription factors in each of the apicomplexans are presented in Additional File 1.

\section{Transcription factor binding sites}

The G-box position weight matrix (PWM) was constructed from the original sequences discovered by Militello and colleagues [22]. The sporozoite and cell invasion PWMs were constructed from the sequences used to generate the $P$. falciparum versions of the respective motifs from earlier work from our lab [5]. In their paper [23], Yuda and colleagues did not provide the raw individual ookinete binding sites necessary for constructing a PWM. Consequently, we ran the motif finder MEME (version 4.2.0) [37] on the upstream regions of the ookinete genes reported by Yuda et al. to identify the ookinete PWM.

\section{Expected regulons}

The sporozoite regulon consisted of a set of genes maximally expressed in sporozoites as determined in previous publications [30,38]. The ribosomal regulon consisted of a set of genes annotated as being part of the ribosome (GO:0005840) using Gene Ontology annotation obtained from PlasmoDB on 10/19/2008. As the Gene Ontology has very few P. falciparum genes annotated as being involved in cell invasion, the cell invasion regulon consisted of genes obtained from an integrated clustering of Gene Ontology terms and expression data [39]. The ookinete regulon consisted of the set of genes found to be maximally expressed in ookinetes extracted from the study by Vontas and colleagues [40].

\section{Assessment of binding site conservation}

The best scoring instance of a particular binding site in the region $2000 \mathrm{bp}$ upstream of each gene (or up until the nearest upstream gene if that was closer) in a genome of interest was identified using PWM_SCAN with the 'stage 2' option which uses the percentile of the observed score between the minimum and maximum achievable by the matrix as a hit score [41]. The average length of intergenic regions in the five Plasmodium species considered is $1476.58 \mathrm{bp}$ (computed from data in $[42,43]$ ). By limiting our analyses to a maximum upstream length of $2000 \mathrm{bp}$, on average, we capture most of the entire intergenic regions in these species and give some extra allowance for longer upstream regions. Given that most of the binding sites were originally discovered in Plasmodium species and also for consistency we anchored the maximum lengths of upstream regions in non-Plasmodium species at $2000 \mathrm{bp}$ as well.

Table 1 Apicomplexan genomes utilized in this paper

\begin{tabular}{|c|c|c|c|c|c|c|c|}
\hline Abbr. & Species & Version & Genome Status & Source & Primary reference & Number of genes & $\begin{array}{l}\text { Number of available } \\
\text { upstream regions }\end{array}$ \\
\hline$C p$ & Cryptosporidium parvum & $02 / 23 / 2007$ & $13 X$ & CryptoDB & {$[44]$} & 3886 & 3875 \\
\hline$\overline{T g}$ & Toxoplasma gondii & $07 / 23 / 2008$ & & ToxoDB & & 8155 & 8120 \\
\hline $\mathrm{NC}$ & Neospora caninum & $08 / 05 / 2008$ & & ToxoDB & & 5761 & 5746 \\
\hline$\overline{B b}$ & Babesia bovis & $04 / 10 / 2008$ & $8 X$ & GenBank & {$[45]$} & 3671 & 3657 \\
\hline$T p$ & Theileria parva & $07 / 30 / 2005$ & Complete & GenBank & {$[46]$} & 4035 & 4025 \\
\hline$T a$ & Theileria annulata & $07 / 29 / 2005$ & $8 X$ & GeneDB & {$[4]$} & 3793 & 3786 \\
\hline$\overline{P f}$ & Plasmodium falciparum & $06 / 28 / 2007$ & Complete & PlasmoDB & {$[2]$} & 5460 & 5456 \\
\hline$\overline{P k}$ & Plasmodium knowlesi & $02 / 22 / 2007$ & $8 X$ & GeneDB & {$[47]$} & 5161 & 4762 \\
\hline$\overline{P V}$ & Plasmodium vivax & $09 / 11 / 2007$ & Complete & GenBank & {$[5]$} & 5390 & 5342 \\
\hline$\overline{P b}$ & Plasmodium berghei & $02 / 27 / 2006$ & $4 X$ & PlasmoDB & {$[43]$} & 12365 & 10238 \\
\hline$\overline{P y}$ & Plasmodium yoelii yoelii & $09 / 10 / 2002$ & $5 X$ & PlasmoDB & {$[48]$} & 8075 & 7603 \\
\hline
\end{tabular}


To identify a score cutoff for high scoring hits in a particular genome, the position weight matrix (PWM) of each site was permuted 100 times to obtain 100 random copies of that site, each with the same base composition as the original site. These 100 permuted sites were also used to scan the upstream genomic regions. Two distributions were then created, one consisting of the scores of the actual site and the other, a background distribution, consisting of the scores of the all the permuted sites. The score at the top one percentile of distinct scores in the background distribution was used as a cutoff for high confidence hits of the actual site. Next, the overlap between genes with high confidence hits of the site in their upstream regions and the set of genes expected to be regulated by the site was assessed by estimating the probability of obtaining the observed amount of overlap (enrichment) by random chance using the hypergeometric distribution. The above procedure was repeated in each genome of interest for the particular site (using the same permuted instances of the site) and the $p$-values from the hypergeometric distribution were compared across species to assess conservation. The procedure is explained pictorially in Figure 2.

Hypergeometric statistics were computed using the phyper function in the $\mathrm{R}$ statistical language. In computing the hypergeometric statistics we used the total number of predicted genes rather than the total number of available upstream regions as the total set size. Genes on short contigs may not have upstream regions or may have unrealistically short upstream regions. By utilizing the total number of genes as the total set size in the hypergeometric computation we are being overly conservative and improved annotation resulting in new upstream regions may improve $p$-values. We exclude upstream regions less than $10 \mathrm{bp}$ - see Table 1 for total numbers of available upstream regions.

Overlap (enrichment) statistics for the cell invasion, ribosomal/G-box and sporozoite sites are given in Tables S1, S2 and S3 respectively (all in Additional File 3). We also assessed the ribosomal genes for enrichment with the sporozoite site and the TRP-1 site discovered by Van Poppel and colleagues [24]. The overlap statistics for these two sets of analyses are in Tables S4 and S5 respectively in Additional File 3.

Additional file 1: Putative transcription factors in apicomplexan proteomes. This file contains Pfam-predicted transcription factors in the apicomplexan species considered in this paper. It has multiple sheets. Each sheet has two columns - the first of which specifies a DNA-binding domain and the second a protein predicted to contain that domain. The sheets are labelled according to the species abbreviations described in Table 1.

Click here for file

[http://www.biomedcentral.com/content/supplementary/1471-2164-11147-S1.XLS]
Additional file 2: Putative transcription factors with similar orthology profiles to the G-box binding site. This file contains the putative transcription factors whose orthology profiles are similar to that of the G-box. Their DNA-binding domains and correlation to the average expression profile of the putative G-box associated ribosomal genes

(Additional File 4) are also given.

Click here for file

[http://www.biomedcentral.com/content/supplementary/1471-2164-11147-S2.XLS]

Additional file 3: Data used for computing binding site enrichment statistics. Contains labelled supplementary tables presenting the data used to compute the binding site enrichment $p$-values for the various regulons.

Click here for file

[http://www.biomedcentral.com/content/supplementary/1471-2164-11147-S3.PDF ]

Additional file 4: Ribosomal genes with high confidence G-box hits in their $\mathbf{2 0 0 0}$ bp upstream regions. This file contains the ribosomal genes with high confidence G-box hits in their 2000 bp upstream regions (data used to construct Figure 5). The file has two columns - the first specifies gene names and the second states gene annotation obtained from PlasmoDB.

Click here for file

[http://www.biomedcentral.com/content/supplementary/1471-2164-11147-S4.XLS ]

Additional file 5: Orthologs of the putative $P$. falciparum

transcription factors. This file contains orthologs (one species per column) of each the putative P. falciparum transcription factors. 'NA' implies that an ortholog was not detected in a particular species.

Columns are labelled according to the species abbreviations described in Table 1. The two MAL8P1.153 orthologs identified by syntenic

examination are highlighted in red.

Click here for file

[http://www.biomedcentral.com/content/supplementary/1471-2164-11147-S5.XLS]

\section{Acknowledgements}

We thank Omar Harb, Lucia Peixoto, David Roos, Larry Singh and Sridhar Hannenhalli for useful feedback; Deborah Pinney, Wei Li and Vishal Nayak for help in locating various genomic datasets; Chih-Horng Kuo for providing us with a Babesia-including OrthoMCL clustering.

This work was funded by NIH R01 Al058515 to CJS.

The Neospora caninum genome sequencing project was funded by BBSRC (UK) and was sequenced at the Wellcome Trust Sanger Institute (WTSI) in Cambridge (UK) in collaboration with University of Liverpool (UK). We wish to thank the WTSI Pathogen Genomics group and the sequencing operations staff for sequencing and making the annotated version of the assembled Neospora caninum genome sequence data publicly available via GeneDB and ToxoDB.

\section{Author details}

${ }^{1}$ Department of Bioengineering, University of Pennsylvania, 240 Skirkanich Hall, 210 South 33rd Street, Philadelphia, Pennsylvania 19104, USA. ${ }^{2}$ Center for Bioinformatics, University of Pennsylvania, 1420 Blockley Hall, 423 Guardian Drive, Philadelphia, Pennsylvania 19104, USA. ${ }^{3}$ Department of Genetics, School of Medicine, University of Pennsylvania, 415 Curie Boulevard, Philadelphia, Pennsylvania 19104, USA.

\section{Authors' contributions}

KE: conceived and designed analyses, performed analyses and drafted the manuscript. CJS: conceived and designed analyses and assisted in drafting the manuscript. All authors read and approved the final manuscript.

Received: 25 May 2009

Accepted: 3 March 2010 Published: 3 March 2010 


\section{References}

1. Roos DS: Themes and Variations in Apicomplexan Parasite Biology. Science 2005, 309:72-73.

2. Gardner MJ, Hall N, Fung E, White O, Berriman M, et al: Genome sequence of the human malaria parasite Plasmodium falciparum. Nature 2002, 419:498-511.

3. Coulson RM, Hall N, Ouzounis CA: Comparative Genomics of Transcriptional Control in the Human Malaria Parasite Plasmodium falciparum. Genome Res 2004, 14:1548-1554.

4. Pain A, Renauld H, Berriman M, Murphy L, Yeats CA, et al: Genome of the Host-Cell Transforming Parasite Theileria annulata Compared with T. parva. Science 2005, 309:131-133.

5. Carlton JM, Adams JH, Silva JC, Bidwell SL, Lorenzi H, et al: Comparative genomics of the neglected human malaria parasite Plasmodium vivax. Nature 2008, 455:757-763.

6. Balaji S, Babu MM, lyer LM, Aravind L: Discovery of the principal specific transcription factors of Apicomplexa and their implication for the evolution of the AP2-integrase DNA binding domains. Nucl Acids Res 2005, 33:3994-4006.

7. Imamura $\mathrm{H}$, Persampieri $\mathrm{JH}$, Chuang $\mathrm{JH}$ : Sequences conserved by selection across mouse and human malaria species. BMC Genomics 2007, 8:372

8. Guo X, Silva J: Properties of non-coding DNA and identification of putative cis-regulatory elements in Theileria parva. BMC Genomics 2008, 9:582.

9. Mullapudi N, Lancto CA, Abrahamsen MS, Kissinger JC: Identification of putative cis-regulatory elements in Cryptosporidium parvum by de novo pattern finding. BMC Genomics 2007, 8:13

10. King MC, Wilson AC: Evolution at two levels in humans and chimpanzees. Science 1975, 188:107-116.

11. Borneman AR, Gianoulis TA, Zhang ZD, Yu H, Rozowsky J, et al: Divergence of Transcription Factor Binding Sites Across Related Yeast Species. Science 2007, 317:815-819.

12. Odom DT, Dowell RD, Jacobsen ES, Gordon W, Danford TW, et al: Tissuespecific transcriptional regulation has diverged significantly between human and mouse. Nat Genet 2007, 39:730-732.

13. De Silva EK, Gehrke AR, Olszewski K, Leon I, Chahal JS, et al: Specific DNA binding by Apicomplexan AP2 transcription factors. Proceedings of the National Academy of Sciences 2008, 105:8393-8398.

14. Bankier AT, Spriggs HF, Fartmann B, Konfortov BA, Madera M, et al: Integrated Mapping, Chromosomal Sequencing and Sequence Analysis of Cryptosporidium parvum. Genome Res 2003, 13:1787-1799.

15. Ling K, Rajandream M, Rivailler $P$, Ivens A, Yap S, et al: Sequencing and analysis of chromosome 1 of Eimeria tenella reveals a unique segmental organization. Genome Res 2007, 17:311-319.

16. Kuo C, Wares JP, Kissinger JC: The Apicomplexan Whole-Genome Phylogeny: An Analysis of Incongruence among Gene Trees. Mol Biol Evol 2008, 25:2689-2698.

17. Chen F, Mackey AJ, Stoeckert CJ, Roos DS: OrthoMCL-DB: querying a comprehensive multi-species collection of ortholog groups. Nucl Acids Res 2006, 34:D363-D368.

18. Beltrao P, Trinidad JC, Fiedler D, Roguev A, Lim WA, et al: Evolution of Phosphoregulation: Comparison of Phosphorylation Patterns across Yeast Species. PLOS Biol 2009, 7:e1000134.

19. Coulson RMR, Ouzounis CA: The phylogenetic diversity of eukaryotic transcription. Nucl Acids Res 2003, 31:653-660.

20. Lindner SE, De Silva EK, Keck JL, Llinás M: Structural Determinants of DNA Binding by a P. falciparum ApiAP2 Transcriptional Regulator. J Mol Biol 2009, 395:558-567.

21. Voss TS, Kaestli M, Vogel D, Bopp S, Beck H: Identification of nuclear proteins that interact differentially with Plasmodium falciparum var gene promoters. Mol Microbiol 2003, 48:1593-1607

22. Militello KT, Dodge M, Bethke L, Wirth DF: Identification of regulatory elements in the Plasmodium falciparum genome. Mol Biochem Parasitol 2004, 134:75-88.

23. Yuda M, Iwanaga S, Shigenobu S, Mair GR, Janse CJ, et al: Identification of a transcription factor in the mosquito-invasive stage of malaria parasites. Mol Microbiol 2009, 71:1402-1414.

24. Van Poppel NFJ, Welagen J, Vermeulen AN, Schaap D: The Complete Set of Toxoplasma Gondii Ribosomal Protein Genes Contains Two Conserved Promoter Elements. Parasitology 2006, 133:19-31.

25. Young J, Johnson J, Benner C, Yan SF, Chen K, et al: In silico discovery of transcription regulatory elements in Plasmodium falciparum. $B M C$ Genomics 2008, 9:70
26. Tanay A, Regev A, Shamir R: Conservation and evolvability in regulatory networks: the evolution of ribosomal regulation in yeast. Proc Natl Acad Sci USA 2005, 102:7203-7208.

27. Ihmels J, Bergmann S, Gerami-Nejad M, Yanai I, McClellan M, et al: Rewiring of the Yeast Transcriptional Network Through the Evolution of Motif Usage. Science 2005, 309:938-940

28. Menard R, Heussler V, Yuda M, Nussenzweig V: Plasmodium preerythrocytic stages: what's new?. Trends in Parasitology 2008, 24:564-569.

29. Lopez-Bigas N, De S, Teichmann S: Functional protein divergence in the evolution of Homo sapiens. Genome Biology 2008, 9:R33.

30. Essien K, Hannenhalli S, Stoeckert CJ: Computational Analysis of Constraints on Noncoding Regions, Coding Regions and Gene Expression in Relation to Plasmodium Phenotypic Diversity. PLOS ONE 2008, 3:e3122

31. Gasch AP, Moses AM, Chiang DY, Fraser HB, Berardini M, et al: Conservation and Evolution of Cis-Regulatory Systems in Ascomycete Fungi. PLOS Biology 2004, 2:e398.

32. Doniger SW, Fay JC: Frequent gain and loss of functional transcription factor binding sites. PLOS Comput Biol 2007, 3:e99.

33. Moses AM, Pollard DA, Nix DA, lyer VN, Li X, et al: Large-scale turnover of functional transcription factor binding sites in Drosophila. PLoS Comput Biol 2006, 2:e130.

34. Li L, Stoeckert CJ, Roos DS: OrthoMCL: identification of ortholog groups for eukaryotic genomes. Genome Res 2003, 13:2178-2189.

35. Zupicich J, Brenner SE, Skarnes WC: Computational prediction of membrane-tethered transcription factors. Genome Biol 2001, 2 RESEARCH0050-0050.6.

36. Finn RD, Tate J, Mistry J, Coggill PC, Sammut SJ, et al: The Pfam protein families database. Nucleic Acids Res 2008, 36:D281-D288.

37. Bailey $T L$, Elkan C: Fitting a mixture model by expectation maximization to discover motifs in biopolymers. Proc Int Conf Intell Syst Mol Biol 1994, 2:28-36.

38. Jeffares DC, Pain A, Berry A, Cox AV, Stalker J, et al: Genome variation and evolution of the malaria parasite Plasmodium falciparum. Nat Genet 2007, 39:120-125.

39. Zhou Y, Young JA, Santrosyan A, Chen K, Yan SF, et al: In silico gene function prediction using ontology-based pattern identification. Bioinformatics 2005, 21:1237-1245.

40. Vontas J, Siden-Kiamos I, Papagiannakis G, Karras M, Waters AP, et al: Gene expression in Plasmodium berghei ookinetes and early oocysts in a coculture system with mosquito cells. Molecular and Biochemical Parasitology 2005, 139:1-13.

41. Levy $\mathrm{S}$, Hannenhalli S: Identification of transcription factor binding sites in the human genome sequence. Mamm Genome 2002, 13:510-514.

42. Carlton JM, Angiuoli SV, Suh BB, Kooij TW, Pertea M, et al: Genome sequence and comparative analysis of the model rodent malaria parasite Plasmodium yoelii yoelii. Nature 2002, 419:512-519.

43. Hall N, Karras M, Raine JD, Carlton JM, Kooij TWA, et al: A Comprehensive Survey of the Plasmodium Life Cycle by Genomic, Transcriptomic, and Proteomic Analyses. Science 2005, 307:82-86.

44. Abrahamsen MS, Templeton TJ, Enomoto S, Abrahante JE, Zhu G, et al: Complete Genome Sequence of the Apicomplexan, Cryptosporidium parvum. Science 2004, 304:441-445.

45. Brayton KA, Lau AOT, Herndon DR, Hannick L, Kappmeyer LS, et al: Genome sequence of Babesia bovis and comparative analysis of apicomplexan hemoprotozoa. PLoS Pathog 2007, 3:1401-1413.

46. Gardner MJ, Bishop R, Shah T, de Villiers EP, Carlton JM, et al: Genome sequence of Theileria parva, a bovine pathogen that transforms lymphocytes. Science 2005, 309:134-137.

47. Pain A, Bohme U, Berry AE, Mungall K, Finn RD, et al: The genome of the simian and human malaria parasite Plasmodium knowlesi. Nature 2008 455:799-803.

48. Carlton JM, Angiuoli SV, Suh BB, Kooij TW, Pertea M, et al: Genome sequence and comparative analysis of the model rodent malaria parasite Plasmodium yoelii yoelii. Nature 2002, 419:512-519.

doi:10.1186/1471-2164-11-147

Cite this article as: Essien and Stoeckert: Conservation and divergence of known apicomplexan transcriptional regulons. BMC Genomics 2010 11:147. 